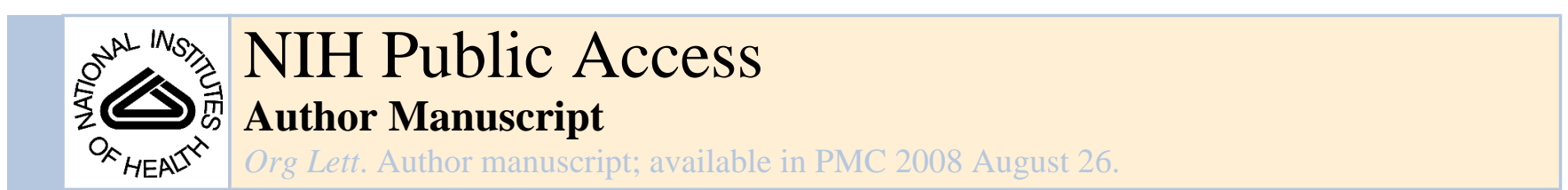

Published in final edited form as:

Org Lett. 2006 April 27; 8(9): 1883-1886. doi:10.1021/ol060458r.

\title{
Synthesis of a Biotin-Derived Alkyne for Pd-Catalyzed Coupling Reactions
}

\author{
Cesear Corona, Bj K. Bryant, and Jeffrey B. Arterburn * \\ New Mexico State University, P.O. Box 30001MSC 3C, Las Cruces, NM 88003
}

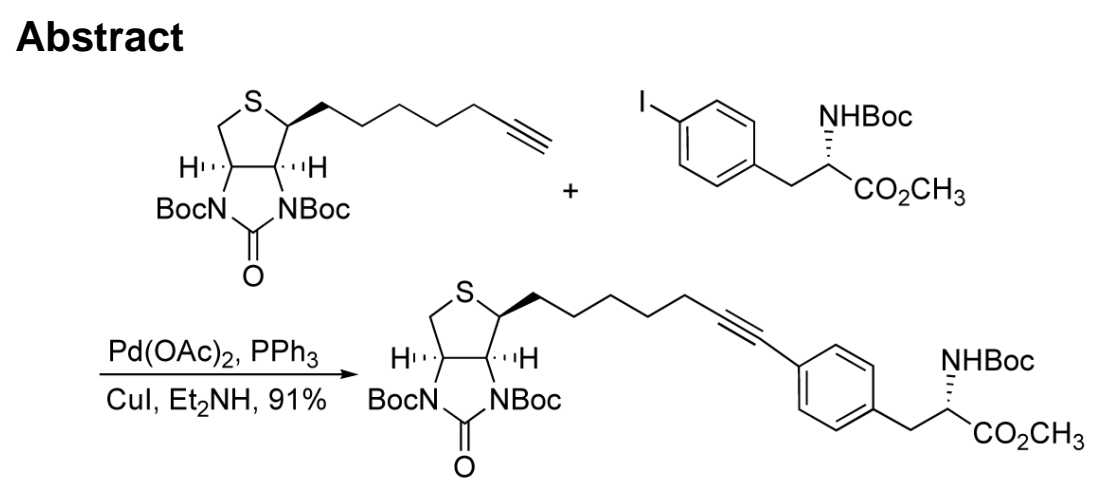

An efficient synthesis of a terminal alkyne derived from $d$-biotin was developed to provide an alternative for carboxylbased biotinylation. This approach was illustrated by the preparation of alkyne- and alkene-linked phenylalanine derivatives using palladium-catalyzed Sonogashira and Oh methodology. (Strept)avidin binding was observed using soluble and immobilized receptors. These results demonstrate the applicability of carbon-linked biotin derivatives for use in affinity-based purification and bioanalytical applications.

The interaction between biotin (Vitamin $\mathrm{H}) \mathbf{1}$ and the glycoprotein avidin is one of the strongest noncovalent associations known $\left(\mathrm{K}_{\mathrm{a}} \sim 2.5 \times 10^{15} \mathrm{M}^{-1}\right)$. Avidin is tetrameric and each subunit is capable of binding a biotin ligand. The high binding affinity and exceptionally slow dissociation rates of biotin results from a network of hydrogen bonds between receptor and the heterocyclic 7-oxo-3-thia-6,8-diazabicyclo[3.3.0]oct-4-yl ligand. The ureido nitrogens of biotin form hydrogen bonds with Thr35 and Asn118, the oxygen contacts Ser16 and Tyr33. Additional hydrogen bonding occurs between the biotin carboxylic acid and avidin residues Ala39, Thr40 and Ser75. The hydrophobic tetrahydrothiophene interacts with Phe79, Trp97, and Trp110. ${ }^{1}$

Avidin and the related tetrameric protein streptavidin share $\sim 33 \%$ conserved amino acids and strong biotin binding affinity $\left(\mathrm{K}_{\mathrm{a}} \sim 1.0 \mathrm{X} 10^{12} \mathrm{M}^{-1}\right){ }^{2}$ Specific interactions include hydrogen bonding between biotin urea nitrogens with Ser 45 and Asp128 and oxygen contacts with Asn23, Ser27 and Tyr43. Interactions also exist between Asn49 and Ser88 and the biotin carboxylic acid group. This network of hydrogen bonding in conjunction with hydrophobic interactions with four Trp residues (Trp 79, 92, 108, 120) and the tetrahydrothiophene result in high binding affinity. ${ }^{3}$

*jarterbu@nmsu.edu

Supporting Information Available: General methods, experimental details, copies of ${ }^{1} \mathrm{H}-\mathrm{NMR}$ and ${ }^{13} \mathrm{C}$-NMR spectra of compounds 1-3, 5-7, 9, 10, 12, 13, and HABA titration curves. 
Biotin-(strept)avidin systems have been used for a variety of applications such as affinity isolation and purification, immunoassay, diagnostics, and localization. $4,5,6$ Nearly any biological entity can be labeled with biotin including peptides, proteins, oligonucleotides and antibodies. Antibody-(strept)avidin conjugates are used in the pretargeting approach to deliver radiolabeled biotin therapeutics and imaging agents. ${ }^{7}$ Other applications include drug delivery 8 and material science. ${ }^{9}$

A variety of biotin-labeling agents are commercially available. ${ }^{10}$ Activated esters $(N$ hydroxysuccinimdyl ester and $p$-nitrophenyl esters), maleimide, and iodoacetyl derivatives are frequently used for coupling to substrates possessing amine or thiol groups. ${ }^{11}$ In vivo applications of amide-linked biotin derivatives can be problematic due to cleavage by the endogenous enzyme biotinidase. ${ }^{12,13}$

Several structural modifications have been described to prevent biotinidase cleavage however, these derivatives exhibit increased dissociation rates from (strept)avidin. Biotin amide derivatives containing a small $\alpha$-substituent have provided the most effective balance of biotinidase stability and (strept)avidin affinity. ${ }^{14}$ Both $N$-methylation of the biotinamide linkage 15 and homologation of the valeric acid chain provided increased resistance to cleavage ${ }^{16}$ as did replacement of the biotinamide connection with thiourea. Unfortunately, increased dissociation rates were observed with all of these linkagemodified derivatives. ${ }^{17}$

We have been interested in developing biotin conjugates of hydrophobic receptor ligands for affinity purification. Available biotin-coupling reagents are not suitable for derivatizing compounds that lack reactive functional groups such as amine, carboxylate or thiol. The installation of polar substituents on a hydrophobic receptor substrate is possible but may decrease receptor-binding affinity due to unfavorable electrostatic interactions, modified solvation characteristics, different lipophilicity (as measured by log P octanol/water), and increased steric interactions. ${ }^{18}$ As an alternative, we considered using non-polar linkages synthesized via catalytic C-C bond coupling methodology. Replacement of the biotin carboxamide with an alkyne or alkene connection would eliminate chemical or enzymatic hydrolysis. The success of this approach depends on the resulting C-C linked biotin substrates maintaining (strept)avidin affinity.

We elected to replace the carboxylic acid group with an alkyne that would allow entry into a wide variety of metal-catalyzed coupling procedures. ${ }^{19}$ The synthesis was initiated by acid catalyzed esterification of biotin. Selective reduction of the ester $\mathbf{2}$ was accomplished using diisobutylaluminum hydride (DIBAL) at $-78{ }^{\circ} \mathrm{C}$, affording alcohol 3 in $96 \%$ yield. Reaction of 3 with toluenesulfonyl chloride in pyridine at $0{ }^{\circ} \mathrm{C}$ provided the sulfonate ester $\mathbf{4}$ in $94 \%$ yield. ${ }^{20}$ The iodide $\mathbf{5 a}$ and bromide $\mathbf{5 b}$ were prepared by halide substitution of $\mathbf{4}$.

Low yields of the desired alkyne $\mathbf{6}$ were obtained from direct lithium acetylide substitution reactions of tosylate $\mathbf{4}$ or iodide $\mathbf{5 a}$. Fortunately, the alkyl bromide $\mathbf{5 b}$ underwent efficient displacement with lithium acetylideethylenediamine in DMSO with careful temperature control $\leq 15^{\circ} \mathrm{C}$ and produced the desired alkyne 6 in high yield. This synthesis provided alkynebiotin derivative 6 with a combined yield of $66 \%$ over five steps from biotin.

We evaluated avidin binding of $\mathbf{6}$ in solution. Competitive displacement of 2-(4'hydroxyphenylazo)benzoic acid (HABA) with biotin derivatives provides a convenient spectroscopic method for assessing avidin binding. In solution, HABA forms a complex with the biotin-binding site of avidin that is characterized by an absorbance band at $500 \mathrm{~nm}$. Displacement of the HABA substrate by biotin results in decreased absorbance at $500 \mathrm{~nm}$. This method has been widely used as a qualitative assay for evaluation of biotinylated substrates. 21 
We constructed a standardized biotin response curve for the HABA-avidin complex in $0.1 \mathrm{M}$ phosphate buffer solution. The decrease in absorbance accompanying additions of $5 \mu \mathrm{L}$ aliquots of $d$-biotin reference solution $\left(5.0 \times 10^{-4} \mathrm{M}\right.$ in $\left.0.10 \mathrm{M} \mathrm{NaH}_{2} \mathrm{PO}_{4}\right)$ were measured in triplicate and plotted against the concentration of added biotin. A response curve was generated analogously for alkyne 6 by addition to the standardized HABA-avidin solution. A decrease in absorbance was observed with the addition of $\mathbf{6}$ demonstrating HABA displacement. From the results of this assay it can be concluded that the association constant of 6 remained high since $\operatorname{HABA}\left(\mathrm{K}_{\mathrm{a}}=6 \times 10^{6} \mathrm{M}^{-1}\right)$ is displaced. ${ }^{21}$ While the HABA displacement was attenuated relative to biotin, effective binding of $\mathbf{6}$ was retained despite replacement of the carboxyl group with alkyne.

Encouraged by the affinity exhibited by $\mathbf{6}$, we proceeded to synthesize a phenylalanine biotin derivative. Amino acids labeled with biotin are important in peptide synthesis. $8,22,23$ Sonogashira cross coupling reactions have been used to produce alkynyl phenylalanine derivatives. ${ }^{24,25}$ In order to increase the solubility of 6 in organic solvents, we protected the urea as the di-tert-butyl carbamate 7. The 4-iodo-phenylalanine derivative 8 was coupled with alkyne 7 under standard conditions using catalytic $\mathrm{Pd}(\mathrm{OAc})_{2}, \mathrm{PPh}_{3}$, and $\mathrm{CuI}$ in diethylamine to give conjugate 9 in $91 \%$ (Scheme 2).

Hydrolysis of the ester with $\mathrm{LiOH}$ (aq.) in $\mathrm{MeOH} / \mathrm{THF}$ at $0{ }^{\circ} \mathrm{C}$, followed by deprotection of the ${ }^{t}$ Boc-groups with trifluoroacetic acid (TFA) in $\mathrm{CH}_{2} \mathrm{Cl}_{2}$ gave the alkynelinked phenylalanine conjugate 10. Spectroscopic characterization by NMR and HPLC-MS confirmed product identity.

We wanted to investigate the affect of shortening the linkage between the biotin heterocycle and the phenylalanine on avidin affinity. Recently, Oh et al. reported a palladium-catalyzed insertion of arylboronic acids into terminal alkynes. ${ }^{26}$ Following this procedure, alkyne 7 was coupled with protected 4-boronic acid derivative $\mathbf{1 1}$ to afford alkene $\mathbf{1 2}$ in $73 \%$ yield (Scheme $3)$. The alkene protons appeared as doublets at $\delta 5.21$ and $5.01(J=1.3 \mathrm{~Hz})$ in the ${ }^{1} \mathrm{H}-\mathrm{NMR}$ spectrum, consistent with the expected alkene. No other alkene isomers were observed by ${ }^{1} \mathrm{H}$ NMR.

The ester 12 was hydrolyzed with $\mathrm{LiOH}$, followed by ${ }^{t} \mathrm{Boc}$-deprotection with TFA/ $\mathrm{CH}_{2} \mathrm{Cl}_{2}$. The product was characterized spectroscopically and by HPLC-MS. The exocyclic alkene isomerized to the more substituted (E)-alkene $\mathbf{1 3}$ during deprotection. The vinyl proton signal was observed as a triplet at $\delta 5.78(J=7.1 \mathrm{~Hz})$ and the allylic methyl group appeared as a singlet at $\delta 1.96$.

The avidin affinities of $\mathbf{1 0}$ and $\mathbf{1 3}$ were evaluated using the HABA assay as described for $\mathbf{6}$. Both derivatives displaced HABA from avidin with comparable efficiency. The $\mathrm{sp}^{2}$ hybridized carbon in alkene $\mathbf{1 3}$ corresponds to the position of the carboxyl in biotinamide derivatives and can be considered to be isosteric although not capable of hydrogen bonding. The linear alkyne connection in $\mathbf{1 0}$ is comparable to the overall length of homologated biotinamide conjugates. These minor differences in length and geometry did not significantly alter the observed binding affinity of $\mathbf{1 0}$ and 13. These derivatives exhibited decreased affinity for avidin relative to biotin as expected from the deletion of the carboxyl group.

Solid-supported streptavidin sorbents have been widely used for batch affinity isolation and purification of biotinylated substrates. Non-binding components are easily removed by elution and isolation of biotinylated substrates is accomplished by dissociation from the supportedstreptavidin. ${ }^{27}$ The strong biotin-streptavidin interaction presents a problem in these applications because harsh conditions are required to dissociate the biotin from the supportmatrix. Biotin derivatives with reduced binding affinity such as desthiobiotin and 2imunobiotin are advantageous because elution occurs with milder conditions. We prepared the 
fluorescent alkynelinked phenylalanine $\mathbf{1 5}$ to evaluate the affinity for immobilized streptavidin (Scheme 4).

The $\alpha$-amino group of $\mathbf{1 0}$ was coupled with Alexafluor 546® succinimidyl ester $\mathbf{1 4}$ to give 15. Compound 15 was dissolved in $0.1 \mathrm{M}$ phosphate buffer $\mathrm{pH} 7.2$ at a concentration of 3.27 $\times 10^{-6} \mathrm{M}$. A $3 \mathrm{~mL}$ aliquot (50\% of the manufacture's recommended loading) was added to centrifuge tube containing strepavidin $(5 \mathrm{~mL})$ on agarose support. The tube was agitated for $15 \mathrm{~min}$, centrifuged for $5 \mathrm{~min}$, and the supernate containing unbound $\mathbf{1 5}$ was isolated. The tube was washed with 10 bed volumes of water to collect unbound substrate $\mathbf{1 5}$. The concentration of free $\mathbf{1 5}$ was determined spectroscopically. The immobilized streptavidin retained $84 \%$ of 15, confirming the potential for C-linked derivatives of $\mathbf{6}$ in batch isolation methods.

The avidin binding of $\mathbf{1 5}$ was studied by fluorescence titration. It was observed that $\mathbf{1 5}$ displayed higher emission intensity in the presence of avidin. Lo et al. have attributed the observed fluorescence enhancement to greater hydrophobicity within the binding pocket. A standardized solution of solubilized avidin was prepared and titrated with $\mathbf{1 5}$. Fluorescence emission enhancement was monitored to determine the concentration required to saturate the receptor. ${ }^{28}$ From this titration experiment, a dissociation constant $\left(\mathrm{K}_{\mathrm{d}}\right)$ of ca. $3.8 \times 10^{-9} \mathrm{M}$ was determined for avidin bound 15 using the method described by Srivastava et al. ${ }^{29}$ This dissociation constant lies between the values of desthiobiotin $\left(\mathrm{K}_{\mathrm{d}}=5.0 \times 10^{-13} \mathrm{M}\right)^{30}$ and 2imunobiotin $\left(\mathrm{K}_{\mathrm{d}}=8.0 \times 10^{-6} \mathrm{M}\right)^{31}$ and is also comparable to other recently reported biotinfluorophore conjugates. 28,32

This study evaluated the potential for carbon-linked biotin conjugates in affinity-based methods. Alkyne $\mathbf{6}$ was efficiently synthesized from $d$-biotin and exhibited strong binding in the HABA assay despite the deletion of the carboxylic acid. The ${ }^{t}$ Boc-protected alkyne 7 was effectively coupled using palladium-catalyzed reactions. Synthetic phenylalanine derivatives 10, 13, and fluorescent probe 15 exhibited affinity for soluble and immobilized (strept)avidin, as expected for binding to the preserved 7-oxo-3-thia-6,8-diazabicyclo[3.3.0]oct-4-yl core. This approach enables conjugation to substrates that cannot be labeled with activated carboxylate derivatives of biotin. The covalent carbon linkage that replaces the carboxamide group of traditional biotin conjugates decreases relative (strept)avidin binding but retains sufficient affinity for bioanalytical applications and provides complete protection from chemical or enzymatic hydrolysis. This approach may be extended to other alkyne coupling and cycloaddition procedures.

\section{Supplementary Material}

Refer to Web version on PubMed Central for supplementary material.

\section{Acknowledgment}

This research was supported by NIH/SCORE GM08136. C. C. was supported by NIH/RISE GM61222. Instrumentation facility was funded by NIH RR16480 from the NCRR-INBRE Program.

\section{References}

(1). Puglise L, Coda A, Malcovati M, Bolognesi M. J. Mol. Biol 1993;231:698. [PubMed: 8515446]

(2). Pahler A, Hendrickson WA, Kolks MAG, Argarana CE, Cantor CR. J. Biol. Chem 1987;29:13933. [PubMed: 3654648]

(3)(a). Chilkoti A, Stayton PS. J. Am. Chem. Soc 1995;117:10622. (b) Klumb LA, Chu V, Stayton PS. Biochemistry 1998;37:7657. [PubMed: 9601024]

(4). Wilchek M, Bayer EA. Anal. Biochem 1988;171:1. [PubMed: 3044183]

(5). Diammandis EP, Christopoulos TK. Clin. Chem 1991;35:625. 
(6). Diamandis, EP.; Christopoulos, TK., editors. Immunoassay. Acedemic Press; San Diego, CA: 1996.

(7). Boerman OC, van Schaijk FG, Oyen WJG, Corstens FHM. J. Nuc. Med 2003;44:400.

(8). Bhunilya S, Park SM, Kim BH. Org. Lett 2005;7:1741. [PubMed: 15844895]

(9). Niemeyer CM. Angew. Chem. Int. Ed 2001;40:4128.

(10). Haugland, RP. Chapter 4. In: Spence, MTZ., editor. The Handbook: A Guide to Fluorescent Probes and Labeling Technologies. 10th. Invitrogen; Eugene, OR: 2005. p. 141

(11). Foulon CF, Alston KL, Zalutsky MR. Bioconjugate Chem 1997;8:179.

(12). Singh R, Maloney EK. Anal. Biochem 2002;304:147. [PubMed: 12009690]

(13). Bogusiewicz A, Mock NI, Mock DM. Anal. Biochem 2004;331:260. [PubMed: 15265731]

(14). Wilbur DS, Hamlin DK, Chyan M, Kegley BB, Pathre PM. Bioconjugate Chem 2001;12:616.

(15). Pazy Y, Kulik T, Bayer EA, Wilchek M, Livnah O. J. Bio. Chem 2002;277:30892. [PubMed: 12055191]

(16). Wilbur DS, Hamlin DK, Pathare PM. Bioconjugate Chem 1997;8:572.

(17). Wilbur DS, Chyan M, Pathare PM, Hamlin DK. Bioconjugate Chem 2000;11:569.

(18). Schneider C, Scholer HF, Schneider RJ. Steroids 2004;69:245. [PubMed: 15183690]

(19). Negishi E, Anastasia L. Chem. Rev 2003;103:1979. [PubMed: 12744698]

(20). DeLaLuz PJ, Golinski M, Watt DS, Vanaman TC. Bioconjugate Chem 1995;6:558.

(21). Hermanson, GT. Bioconjugate Techniques. Academic Press; San Diego, CA: 1996. p. 591

(22). Gallivan JP, Lester HA, Dougherty DA. Chem. Biol 1997;4:739. [PubMed: 9375252]

(23). Kumar V, Aldrich JV. Org. Lett 2003;5:613. [PubMed: 12605472]

(24). Sonogashira K, Tohda Y, Hagihara N. Tetrahedron Lett 1975;50:4467.

(25). Hoffmanns U, Metzler-Nolte N. Bioconjugate Chem 2006;17:204.

(26). Oh CH, Jung HH, Kim KS, Kim N. Angew. Chem. Int. Ed 2003;42:805.

(27). Streptavidin Agarose: For Affinity Chromatography and Immunopecipitation of Biotinylated Proteins. Invitrogen; Eugene, OR: 2003. Techincal Document \#25-0524.

(28)(a). Lo KK, Hui W, Ng DC. J. Am. Chem. Soc 2002;124:9344. [PubMed: 12167008] (b) Lo KK, Hui W. Inorg. Chem 2005;44:1992. [PubMed: 15762726]

(29). Wang Z, Kumar NR, Srivastava DK. Anal. Biohem 1992;206:376.

(30). Muller W, Ringsdorf H, Rump E, Wildberg G, Zhang X, Angermaier L, Knoll W, Liley M, Spinke J. Science 1993;262:1706. [PubMed: 8259513]

(31). Reznik GO, Vajda S, Sano T, Cantor CR. Proc. Natl. Acad. Sci. U. S. A 1998;95:13525. [PubMed: 9811833]

(32). Marek M, Kaiser K, Gruber HJ. Bioconjugate Chem 1997;8:560. (b) Lo KK, Lee TK. Inorg. Chem 2004;43:5275. [PubMed: 15310205]

Org Lett. Author manuscript; available in PMC 2008 August 26. 

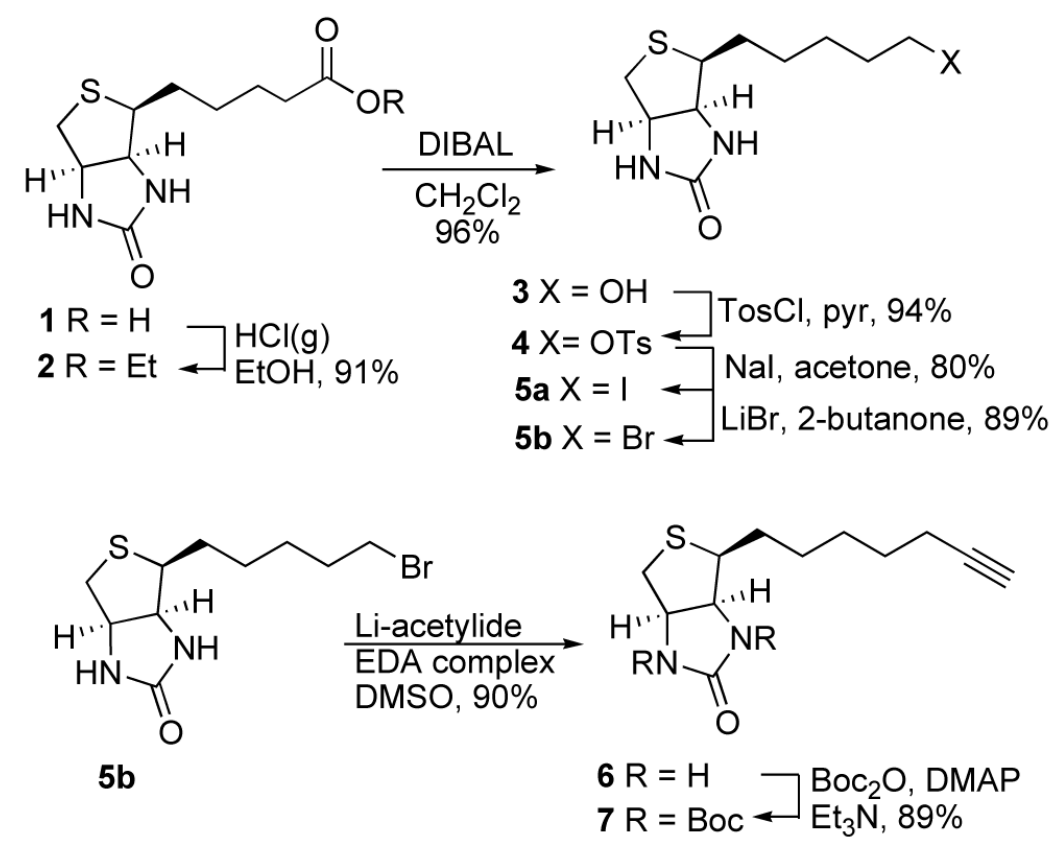

Scheme 1.

Synthesis of Biotin-Alkyne 

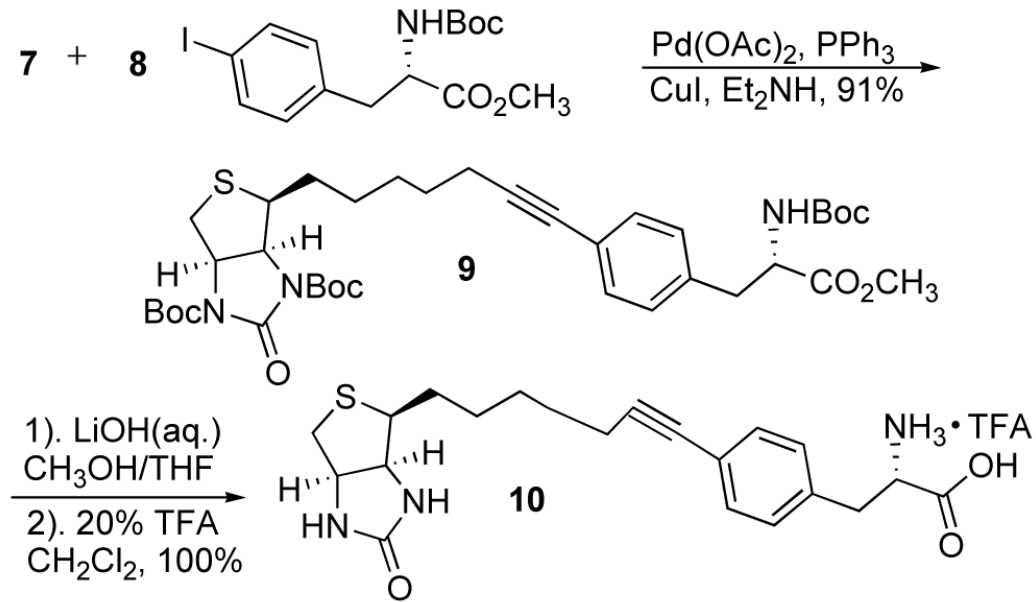

Scheme 2.

Synthesis of alkyne derivatives. 

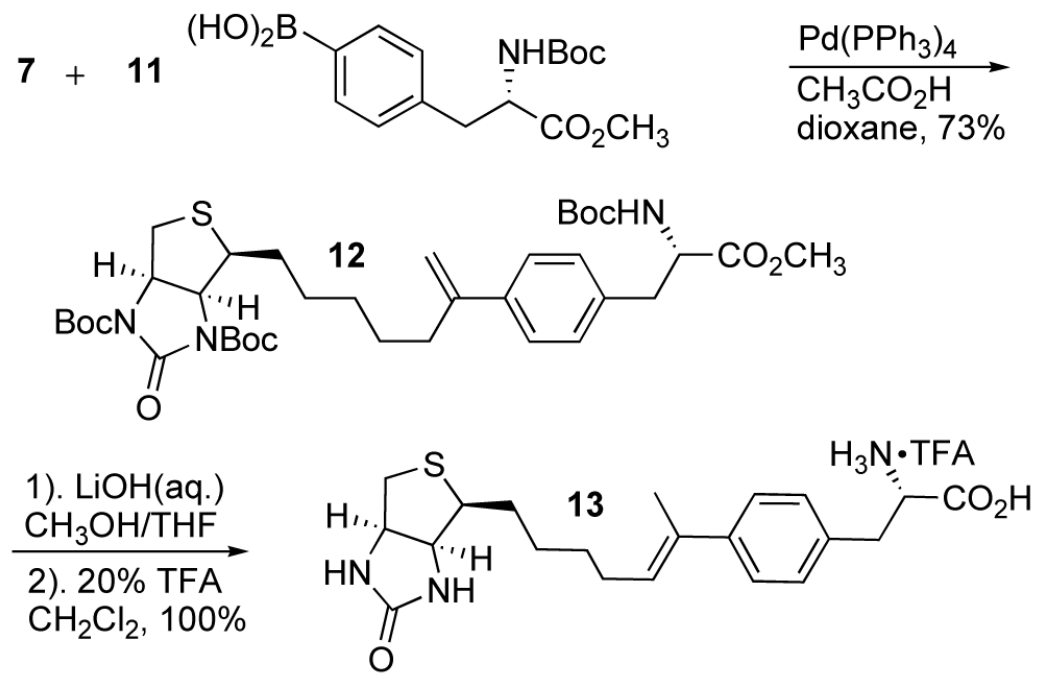

Scheme 3.

Synthesis of Alkene Derivatives. 


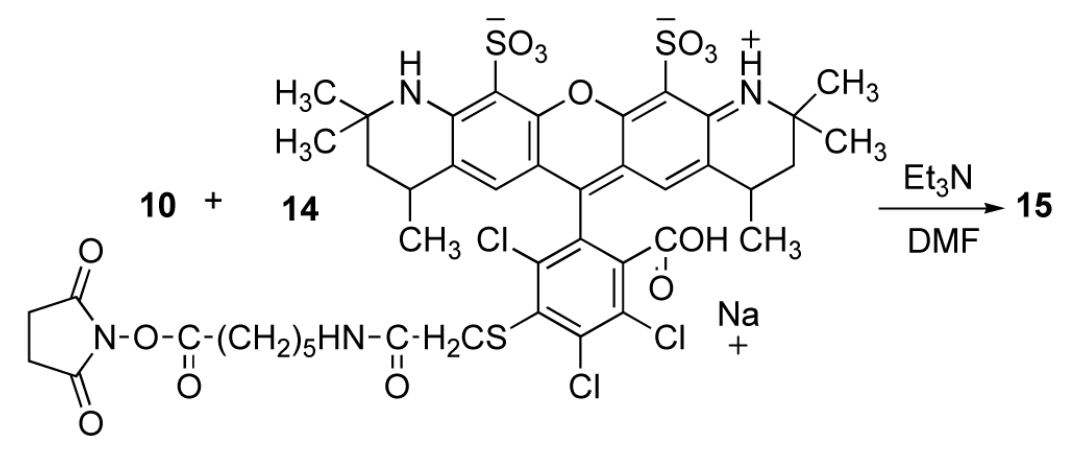

Scheme 4.

Synthesis of Biotin Alexafluor ${ }^{\circledR}$ conjugate. 


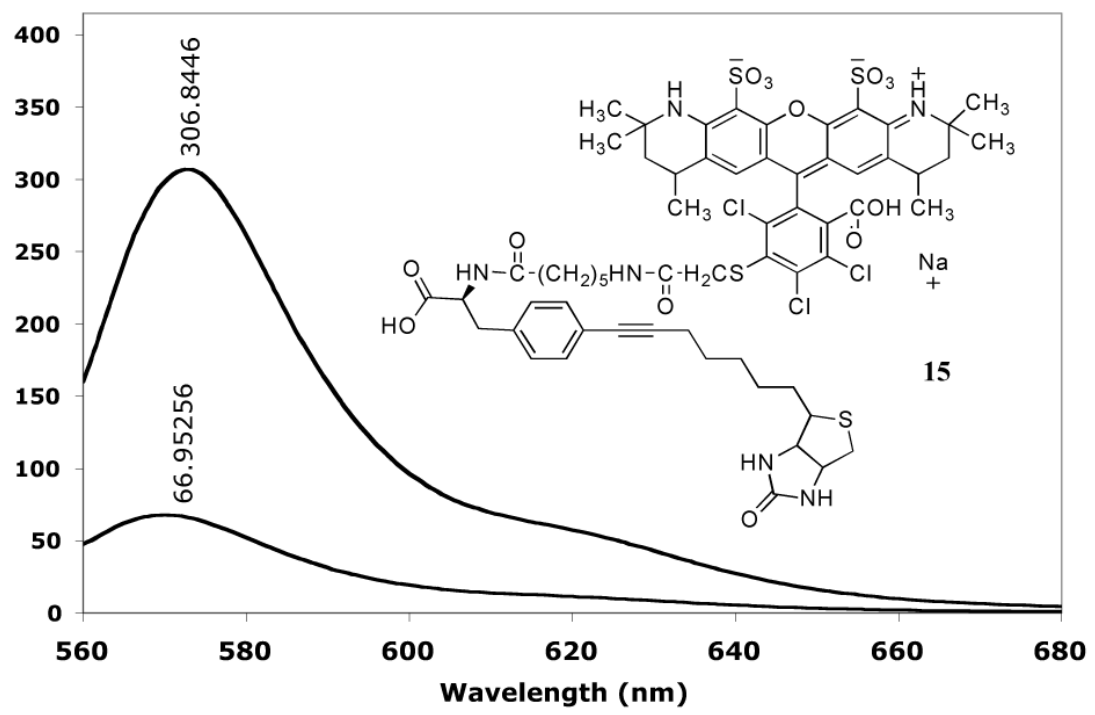

Figure 1.

Emission Spectrum of $\mathbf{1 5 .}$ 